\title{
ECG-based atrial fibrillation detection using different orderings of Conjugate Symmetric-Complex Hadamard Transform
}

\author{
Ambika Annavarapu, Padmavathi Kora * \\ ECE, GRIET, India
}

\section{A R T I C L E I N F O}

\section{Article history:}

Received 1 June 2016

Received in revised form 4 August 2016

Accepted 4 August 2016

Available online 11 August 2016

\section{Keywords:}

Atrial Fibrillation

ECG

CS-SCHT

Neural network classifier

\begin{abstract}
A B S T R A C T
Atrial Fibrillation (AF) is a classification of cardiac disrhythmia is an arrhythmia in which the heartbeat is irregular, too fast, or too slow. Because of this erratically changing behavior, effective pumping of blood by the heart to other organs results in malfunctioning of them. Generally, AF is seen commonly in elder people who are suffering from heart failure. To effectively treat AF, automatic detection methods based on electrocardiograph (ECG) monitoring is highly desirable. The objective of this study is to develop a novel algorithm able to detect atrial fibrillation episodes supervising a standard superficial ECG lead. In this discussion, AF is detected by considering the MIT/BIH arrhythmia database. The features of this database is extracted by using the different orderings of Conjugate Symmetric-Complex Hadamard Transform (CS-CHT), namely, natural order, Paley order, sequency order, and Cal-Sal order as they are fast and can be implemented with less memory usage as compared with the previous techniques in literature. The results obtained are applied to Levenberg-Marquardt Neural Network (LM NN) classifier and the performances of these techniques were estimated in terms of sensitivity, specificity, and overall detection accuracy on the datasets.

(c) 2016 The Society of Cardiovascular Academy. Production and hosting by Elsevier B.V. All rights reserved. This is an open access article under the CC BY-NC-ND license (http://creativecommons.org/licenses/by-nc-nd/4.0/).
\end{abstract}

\section{Introduction}

AF is an arrhythmia due to the anomalous discharges of electrical signals in the atria of heart in comparison to the ventricles, leading to the potential stroke due to the clot formation in the atrium. Effective prevention of $\mathrm{AF}^{2,5}$ is possible by detecting the heart activities through analysis of electrocardiogram (ECG) ${ }^{16}$ Episodes of AF comprise of unbalanced heart beat interval (RR) and/or low P-wave amplitude in the QRS complex in addition to the irregular cardiac frequency. In this paper, detection of AF is carried out by the extraction of atrial activity of MIT/BIH arrhythmia database. Primarily, noise in the signal is removed using filters and then the non-uniform beats obtained are converted to uniform beats of size 128 by resampling in order to extract features of the atrial with non-linear signal processing technique of 12-lead ECG with the detection of the spectral peak detection and spectral entropy by applying Fourier transforms in order to avoid the atrial and ventricular spectrum overlapping, which cannot be removed by linear filtering. But the previous works ${ }^{4}$ failed to achieve this detection. In this paper, this drawback in feature extraction is overcome by considering different orders of Conjugate Symmetric-Complex Hadamard Transform (CS-CHT), ${ }^{3}$ namely, natural order, Paley or dyadic order, symmetric order, and Cal-Sal order. ${ }^{6,1}$ These extracted features are

\footnotetext{
* Corresponding author.

E-mail addresses: ambika@griet.in (A. Annavarapu), Padma386@gmail.com (P. Kora).

Peer review under responsibility of The Society of Cardiovascular Academy.
}

categorized into two classes, namely, Normal signal and AF signal by applying to a Levenberg-Marquardt Neural Network (LM NN) classifier. The optimized technique from all the techniques from different orders of CS-CHT is obtained by considering the sensitivity and specificity plot.

\section{System Modeling}

The detection of AF signal, ${ }^{9,10}$ as shown in Fig. 1. using the electrocardiograph method, is performed in this paper by considering the following processes:

\section{A. Preprocessing}

The preprocessing contains mainly three steps

- Removing noise

- Segmenting the ECG files into beats

- Converting the non-uniform beats into uniform

The noise from the ECG signal is removed by using the filters. The obtained ECG files are segmented into beats by detecting the "R" peaks. The samples obtained from these beats are non-uniform samples. By using a technique called resampling, uniform samples of size 200 are generated from these non-uniform samples in each beat. The MIT-BIH Record numbers of AF database of 26 patients and Normal Sinus 


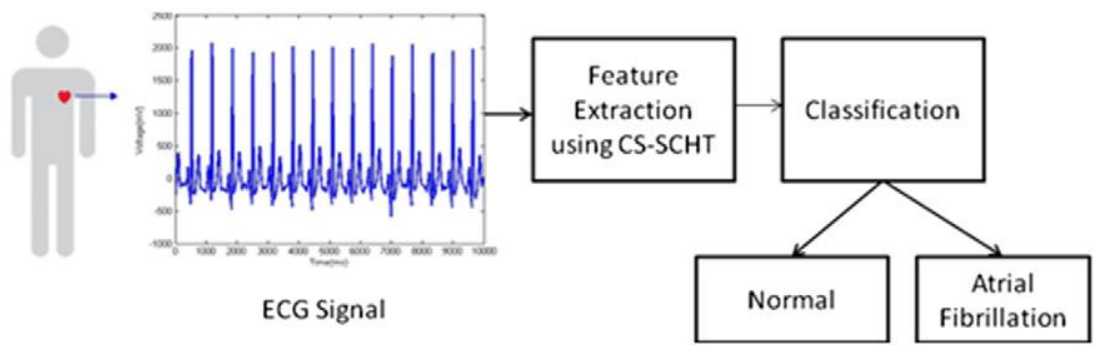

Fig. 1. ECG classification.

Rhythm database of 18 patients are used to detect AF. The sampling rate of normal signals is $128 \mathrm{~Hz}$ and AF signals are $250 \mathrm{~Hz}$. For easy processing, they both are resampled again with the same sampling rate. De-noising of ECG signal is applied to Sgolay FIR smoothing filter. Beat is the $(2 / 3)^{r d s}$ of the RR interval to the right of the R peak and (1/ $3)^{r d s}$ of the RR interval to the left of the R peak. After segmentation, each beat is resampled to 200 samples.

\section{B. Feature Extraction}

In real-time applications, Hadamard transform is used for the signals to be processed using various techniques like signal and image processing, digital logics because of uncomplicated realization of the fast algorithm. But Hadamard transform cannot be applied to complex signals, so, complex Hadamard transform is implemented, which consists of $\{ \pm 1, \pm \sqrt{-1}\}$. The memory requirement for these transforms is high as compared to the DFT because DFT depends on frequency but not on sequency. Hence, a Conjugate Symmetric-Complex Hadamard Transform (CS-CHT) is introduced to reduce this memory requirement. Hadamard matrices can be ordered in different methods namely, natural, dyadic or Paley or bit-reversed, sequency, ${ }^{8}$ and cal-sal orderings. ${ }^{7}$

By using the tensorial products, the rows of a natural order CSComplex Hadamard matrices are obtained as represented below in Eq. (1).

$H_{S}=\left[\begin{array}{cc}H_{S / 2} & H_{S / 2} \\ H_{S / 2}^{\prime} T_{S / 2} & -H_{S / 2}^{\prime} T_{S / 2}\end{array}\right]$

where $T_{S}$ and $H_{S / 2}$ are represented using Eqs. (2) and (3).

$T_{S}=\left[\begin{array}{cc}I_{S / 2} & 0 \\ 0 & j I_{S / 2}\end{array}\right]$

$H_{S / 2}^{\prime}=\left[\begin{array}{cc}H_{S / 4}^{\prime} & H_{S / 4}^{\prime} \\ H_{S / 4}^{\prime} I_{S / 4}^{\prime} & -H_{S / 4}^{\prime} I_{S / 4}^{\prime}\end{array}\right]$

where $I_{S / 2}^{\prime}$ is defined using Eq. (4).

$\mathrm{I}_{S / 2}^{\prime}=\left[\begin{array}{cc}I_{S / 4} & 0 \\ 0 & -I_{S / 4}\end{array}\right]$

By placing $S=2$ in Eq. (1), we have

$H_{S}=\left[\begin{array}{cc}1 & 1 \\ 1 & -1\end{array}\right]$

CS-Complex Hadamard matrices in natural order satisfy the Good's theorem, which states the uniformity between the tensorial product and normal product with the sparse matrix as represented in Eq. (5).

$\left(H_{S}\right)_{n a t}=\left[\begin{array}{cccccccc}1 & 1 & 1 & 1 & 1 & 1 & 1 & 1 \\ 1 & -1 & 1 & -1 & 1 & -1 & 1 & -1 \\ 1 & j & -1 & -1 & 1 & j & -1 & -j \\ 1 & -j & -1 & j & 1 & -j & -1 & j \\ 1 & 1 & j & j & -1 & -1 & -j & -j \\ 1 & -1 & j & -j & -1 & 1 & -j & j \\ 1 & -1 & -j & j & -1 & 1 & j & -j \\ 1 & 1 & -j & -j & -1 & -1 & j & j\end{array}\right]$

A CS-Complex Hadamard matrix is said to be in Paley ordered if the Rademacher functions are used to generate the Hadamard matrix. A Paley-ordered matrix is obtained from the natural ordered matrix by simply changing the row of a natural order with the bit-reverse of the row number. For example, for an $8 \times 8$ matrix, the natural row number 3 (011) is replaced by 6 (110). The FFT is similar to the fast algorithms of CS-Complex Hadamard matrix. The Paley ordered CS-Complex Hadamard matrix is represented in Eq. (6).

$\begin{aligned}\left(H_{S}\right)_{p a l} & =\left[\begin{array}{cccccccc}1 & 1 & 1 & 1 & 1 & 1 & 1 & 1 \\ 1 & 1 & j & j & -1 & -1 & -j & -j \\ 1 & j & -1 & -j & 1 & j & -1 & -j \\ 1 & -1 & -j & j & -1 & 1 & j & -j \\ 1 & -1 & 1 & -1 & 1 & -1 & 1 & -1 \\ 1 & -1 & j & -j & -1 & 1 & -j & j \\ 1 & -j & -1 & j & 1 & -j & -1 & j \\ 1 & 1 & -j & -j & -1 & -1 & j & j\end{array}\right] \\ \left(\mathrm{H}_{\mathrm{S}}\right)_{\text {seq }} & =\left[\begin{array}{cccccccc}1 & 1 & 1 & 1 & 1 & 1 & 1 & 1 \\ 1 & 1 & j & j & -1 & -1 & -j & -j \\ 1 & -1 & -j & j & -1 & 1 & j & -j \\ 1 & j & -1 & -j & 1 & j & -1 & -j \\ 1 & -j & -1 & j & 1 & -j & -1 & j \\ 1 & 1 & -j & -j & -1 & -1 & j & j \\ 1 & -1 & j & -j & -1 & 1 & -j & j \\ 1 & -1 & 1 & -1 & 1 & -1 & 1 & -1\end{array}\right]\end{aligned}$

$\left(H_{S}\right)_{\text {cal-sal }}=\left[\begin{array}{cccccccc}1 & 1 & 1 & 1 & 1 & 1 & 1 & 1 \\ 1 & -1 & -j & j & -1 & 1 & j & j \\ 1 & -j & -1 & j & 1 & -j & -1 & j \\ 1 & -1 & j & -j & -1 & 1 & -j & j \\ 1 & -1 & 1 & -1 & 1 & -1 & 1 & -1 \\ 1 & 1 & -j & -j & -1 & -1 & j & j \\ 1 & j & -1 & -j & 1 & j & -1 & -j \\ 1 & 1 & j & j & -1 & -1 & -j & -j\end{array}\right]$

Real-time applications use an ordering called sequency ordering, which is achieved by incrementing number of zero crossings. The sequency ordering is obtained from Paley ordering by simply applying gray code conversion for a row in Paley ordering. For example, for an $8 \times 8$ matrix, the Paley row number 7 (111) is replaced by 4 (100) in Sequency ordering. Eq. (7) represents the eight-order CS-Complex Hadamard Matrix in sequency order.

Another ordering algorithm proposed for CS-Complex Hadamard Matrix is Cal-Sal ordering. It can be achieved by placing even numbered 
rows in the first half of the matrix in ascending order and odd numbered rows in the second half of the matrix in descending order as represented using Eq. (8). This order is more advantageous that the storage locations required are dropped by half, i.e., $S$ storage locations required are dropped to $S / 2$. Cal-Sal order matrix is symmetric.

\section{Levenberg-Marquardt Neural Network (LMNN) Classifier}

A back propagation Levenberg-Marquardt Neural Network (LMNN) method is used to detect the AF in this paper. Rapid execution of the network is done by using NN. A well-designed NN network comprises of 20 input neurons, 10 neurons in the hidden layer, and 3 neurons in the output layer. A comparison of these results is made with the Scalar Conjugate Gradient Neural Network (SCG NN) in which a conjugate direction search is performed instead of linear search. LMNN is a very simple and robust method for an approximating function. This network training and testing are performed by using 1800 ECG beats and 1006 ECG beats correspondingly by setting the total number of iterations to 1000 and mean square error less than 0.001 with the minimum time requirement.

\section{Results and discussion}

The convergence speed and final accuracy are increased by applying the results obtained from feature extraction as the input of a neural network. A sampling rate of 128 samples/beat is used for resampling process. In this feature, extraction techniques of CS-CHT using different orderings of natural, Paley, sequency, and Cal-Sal are used. The performance of these four techniques is compared as shown in Table 1 below.

For measuring accuracy, two parameters are used as shown in (8). They are sensitivity and specificity as represented in the Eqs. (9) and (10).

specificity $=\frac{\text { True }_{\text {Negative }}}{\text { True }_{\text {Negative }}+\text { False }_{\text {Positive }}} \times 100$

sensitivity $=\frac{\text { True }_{\text {Positive }}}{\text { True }_{\text {Positive }}+\text { False }_{\text {Negative }}} \times 100$

Accuracy $=\frac{T P+T N}{T P+T N+F P+F N} \times 100$

$\operatorname{TP}\left(\right.$ True $\left._{\text {Positive }}\right)=$ Count of all the correctly classified Abnormal beats

TP $\left(\right.$ True $\left._{\text {Negative }}\right)=$ Count of all the correctly classified Normal beats

FP $\left(\right.$ False $\left._{\text {Positive }}\right)=$ Count of Normal beats classified as Normal beats

FN $\left(\right.$ False $\left._{\text {Negative }}\right)=$ Count of Abnormal beats classified as Abnormal beats

The network performance is checked by applying a multilayer NN in training mode to decide if there is any requirement of any changes to the training progression or the data set or the system planning. The performance of different classifiers is compared as shown in Fig. 2. by plotting sensitivity (True Positive $_{\text {rate) }}$ and specificity (False Positive $_{\text {rate) }}$ and it is obtained that the cal-sal order of CS-CHT achieves the maximum performance compared to that of the remaining orders.

Table 1

Classification using LM NN classifier.

\begin{tabular}{lccc}
\hline Classifier & Sensitivity & Specificity & Accuracy \\
\hline$C S-N C H T+L M N N$ & $87.3 \%$ & $89.9 \%$ & $67.8 \%$ \\
$C S-P A L E Y C H T+L M N N$ & $89.2 \%$ & $91.2 \%$ & $89.3 \%$ \\
$C S-S C H T+L M N N$ & $92.2 \%$ & $93.342 \%$ & $93.2 \%$ \\
$C S-C A L-S A L C H T+L M N N$ & $98.7 \%$ & $99.97 \%$ & $99.1 \%$ \\
\hline
\end{tabular}

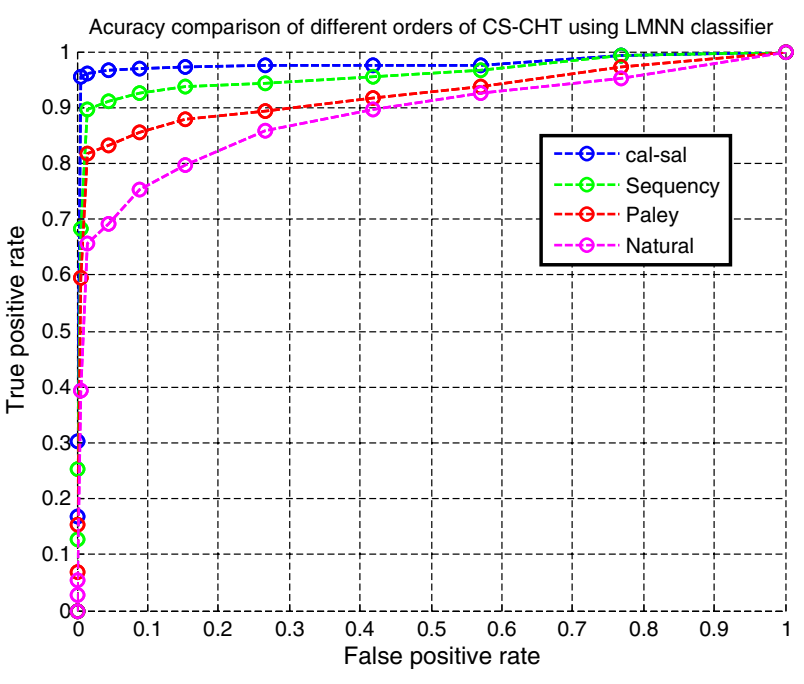

Fig. 2. Comparison of different orders of CS-CHT using LM NN classifier.

The proposed method is compared with the other four detection algorithms as shown in the Table 2 such as, RR interval (RRI), AR coefficients, symbolic dynamics (SD), and Shannon entropy and WTC features in terms of related features selected from the original database and classification accuracy obtained from different classifiers using Matlab software. The work in Ref. 14 explored an experimental study based on the difference between RR intervals for extracting relevant features for the detection of AF. The values of sensitivity and specificity are $94.4 \%$ and $97.2 \%$, respectively. The work presented in Ref. 12 used AR coefficients as features for classification AF using three different classifiers. AR coefficients are calculated for each 15-second data sequence length. The values of specificity and sensitivity are $96.14 \%$ and $93.20 \%$, respectively. The work proposed in Ref. 13 used three statistical methods for the detection of AF. These techniques are tested on AF database and Normal database. The values of sensitivity and specificity are $97.2 \%$ and $95.91 \%$, respectively. The work proposed in Ref 16 used SD and entropy and computed various operations like nonlinear or linear integer filtering. Online analytical processing of the method can be achieved using this novel algorithm. The values of sensitivity, specificity, and accuracy are $96.89 \%, 98.27 \%$, and $98.03 \%$ respectively.

The work proposed in Ref. 11 used WTC coefficients for the detection of AF. The WTC features for the normal and AF datasets are calculated. These features are optimized using PCA algorithm. The values of sensitivity, specificity, and accuracy are $100 \%, 96.9 \%$, and $99.1 \%$, respectively.

From the experiments, this study concludes that the proposed beat feature optimization technique with cal-sal order of CS-CHT outperformed other three algorithms with selection of minimal number of relevant features using CS-CHT. The proposed method shows the highest classification accuracy for the detection of AF. The CS-CHT has been employed intelligently to select the most relevant features that could increase the classification accuracy while ignoring noisy and redundant features.

This procedure helps us to automate a certain section or part of the diagnosis and then it will help the medical community to focus on other crucial sections. This has also increased the accuracy of diagnosis.

\section{Conclusions}

Automation of diagnosis of the human heart by measuring ECG plays a major role in saving a patient. In this paper, this life saviour is achieved in detection of AF signal with an accuracy of $99.9 \%$ by applying CS-SCHT technique to the LMNN classifier. The obtained experimental results have shown that the proposed SCHT method can extract more relevant features than the other methods proposed in the literature with highest classification accuracy for the detection of AF. 
Table 2

Comparative study of detection of AF.

\begin{tabular}{|c|c|c|c|c|}
\hline Studies & Approach & $\begin{array}{l}\text { Sensitivity } \\
(\%)\end{array}$ & $\begin{array}{l}\text { Specificity } \\
(\%)\end{array}$ & $\begin{array}{l}\text { Accuracy } \\
(\%)\end{array}$ \\
\hline $\begin{array}{l}\text { Tateno et al. } \\
(2001)^{14}\end{array}$ & RR interval & 91.20 & 96.08 & - \\
\hline Parvaresh et al. (2011) ${ }^{12}$ & AR coefficients & 96.14 & 93.20 & 90.09 \\
\hline $\begin{array}{l}\text { Lee et al. } \\
(2013)^{13}\end{array}$ & RR interval & 97.26 & 95.91 & - \\
\hline $\begin{array}{l}\text { Zhou et al. } \\
(2014)^{15}\end{array}$ & SD and entropy & 96.89 & 98.27 & 98.03 \\
\hline Padmavathi Kora et al. $(2016)^{11}$ & WTC features & 100 & 96.9 & 99.1 \\
\hline Proposed approach & Cal-sal order of CS-CHT & 99.97 & 98.7 & 99.5 \\
\hline
\end{tabular}

\section{Acknowledgment}

This work was supported by Gokaraju Rangaraju Institute of Engineering \& Technology, Hyderabad, India.

\section{References}

1. Algorithms for converting sequency-, dyadic, and Hadamard ordered Walsh functionsMath Comput Simul 1985:471-477.

2. Bollmann A, Husser D, Mainardi L, et al. Analysis of surface electrocardiograms in atrial fibrillation: techniques, research, and clinical applications. Eur Soc Cardiol 2006 911-926.

3. Aung A, Boon PN, Rahardja S. Conjugate symmetric sequency-ordered complex Hadamard transform. IEEE Trans Sig Process 2009:2582-2593.

4. Cvetkovic D, Übeyli ED, Cosic I. Wavelet transform feature extraction from human PPG, ECG, and EEG signal responses to ELF PEMF exposures: a pilot study. Digit Sig Process 2008;18:861-874.

5. Niu J, Gokana V, Goh S, Lissorgues G, Phua CT. A novel approach to detect atrial fibrillation efficiently and accurately from 48 hours of ECG data.J Electron Commun Eng Res January 2014;2(1):1-6

6. RAO KR, DEVARAJAN V, VLASENKO V, NARASIMHAN MA. CalSal Walsh-Hadamard transform. IEEE 1978:605-607.
7. Kunt M. In place computation of the Hadamard transform in cal-sal order. Sig Process 1979:227-231.

8. Manz JW. A sequency-ordered fast Walsh transform. IEEE Trans Audio Electroacoustics 1972:204-205.

9. Couceiro R, Carvalho P, Henriques J, Antunes M, Harris M, Habetha J. Detection of atrial fibrillation using model-based ECG analysis. IEEE Tampa 2008:1-6.

10. Sahoo SK, Lu W, Teddy SD, Kim D, Feng M. Detection of atrial fibrillation from non-episodic ECG data: a review of methods. Conf Proc IEEE Eng Med Biol Soc 2011 4992-4995.

11. Padmavathi K, Ramakrishna KS. Detection of atrial fibrillation using continuous wavelet transform and wavelet coherence. Int J Syst Control Commun 2015;6(4): 292-304.

12. Parvaresh S, Ayatollahi A. Automatic atrial fibrillation detection using autoregressive modeling. 2011 International Conference on Biomedical Engineering and Technology; 2011.

13. Lee J. Atrial fibrillation detection using an iPhone 4S. Biomed Eng IEEE Trans 2013;60(1):203-206.

14. Tateno K, Glass L. A method for detection of atrial fibrillation using RR intervals. Comput Cardiol 2000:391-394.

15. Zhou X. Automatic online detection of atrial fibrillation based on symbolic dynamics and Shannon entropy. Biomed Eng Online 2014;13(1):18.

16. Kora P. Hybrid Firefly and Particle Swarm Optimization Algorithm for the Detection of Bundle Block. International Journal of Cardiovascular Academy, Elsevier; Dec 2015 